\title{
Phases of the generalized two-leg spin ladder: A view from the SU(4) symmetry
}

\author{
P. Lecheminant \\ Laboratoire de Physique Théorique et Modélisation, CNRS UMR 8089, Université de Cergy-Pontoise, \\ 5 Mail Gay-Lussac, Neuville sur Oise, 95031 Cergy-Pontoise Cedex, France \\ K. Totsuka \\ Yukawa Institute for Theoretical Physics, Kyoto University, Kyoto 606-8502, Japan
}

\begin{abstract}
The zero-temperature phases of a generalized two-leg spin ladder with four-spin exchanges are discussed by means of a low-energy field theory approach starting from an SU(4) quantum critical point. The latter fixed point is shown to be a rich multicritical point which unifies different competing dimerized orders and a scalar chirality phase which breaks spontaneously the time-reversal symmetry. The quantum phase transition between these phases is governed by spin-singlet fluctuations and belongs to the Luttinger universality class due to the existence of an exact U(1) self-duality symmetry.

PACS numbers: 75.10.Jm
\end{abstract}

Multi-spin exchange interactions have attracted much interest over the years both theoretically and experimentally [1]. Recently, ring exchange interactions have been invoked for the description of magnetic properties of spin ladder compound $\mathrm{La}_{x} \mathrm{Ca}_{14-x} \mathrm{Cu}_{24} \mathrm{O}_{41}$ [2] and for their ability to induce new exotic phases in quantum magnetism [3]. In this respect, a scalar chirality phase [4], which breaks spontaneously the time-reversal symmetry, has been found in the two-leg spin$1 / 2$ ladder for a sufficiently strong four-spin cyclic exchange [5, 6]. Such an exotic ground state is, in fact, not specific to this spin ladder and exact ground states with scalar chirality long-range order have been obtained for a wider class of two-leg spin ladders with four-spin interactions [7]. A central question is the determination of all possible ground states stabilized by four-spin exchanges and the elucidation of the nature of the quantum phase transition between these phases. In this letter, we will study a general two-leg spin-1/2 ladder with four-spin exchanges defined by [7]

$$
\begin{aligned}
\mathscr{H}_{\text {gen }} & =J_{1} \sum_{n} \sum_{p=1}^{2} \mathbf{s}_{p, n} \cdot \mathbf{s}_{p, n+1}+J_{\perp} \sum_{n} \mathbf{s}_{1, n} \cdot \mathbf{s}_{2, n} \\
& +J_{d} \sum_{n}\left(\mathbf{s}_{1, n} \cdot \mathbf{s}_{2, n+1}+\mathbf{s}_{1, n+1} \cdot \mathbf{s}_{2, n}\right) \\
& +J_{r r} \sum_{n}\left(\mathbf{s}_{1, n} \cdot \mathbf{s}_{2, n}\right)\left(\mathbf{s}_{1, n+1} \cdot \mathbf{s}_{2, n+1}\right) \\
& +J_{11} \sum_{n}\left(\mathbf{s}_{1, n} \cdot \mathbf{s}_{1, n+1}\right)\left(\mathbf{s}_{2, n} \cdot \mathbf{s}_{2, n+1}\right) \\
& +J_{d d} \sum_{n}\left(\mathbf{s}_{1, n} \cdot \mathbf{s}_{2, n+1}\right)\left(\mathbf{s}_{1, n+1} \cdot \mathbf{s}_{2, n}\right),
\end{aligned}
$$

where $\mathbf{s}_{p, n}$ are spin-1/2 operators at site $n$ on chain $p=1,2$. The Hamiltonian (1) is the most general translation-invariant two-leg spin ladder which (i) consists of SU(2)-symmetric interactions involving two neighboring rungs and (ii) has $\mathbb{Z}_{2}$ invariance under the permutation of the two chains $\mathscr{P}_{12}$. In the following, our strategy is to start from a point with the maximal symmetry in a problem consisting of two spin-1/2 operators, i.e., an SU(4) symmetry. The resulting SU(4) model displays a quantum critical behavior which enables us to develop a low-energy approach to investigate the different $T=0$ gapped phases induced by the $\mathrm{SU}(2) \times \mathbb{Z}_{2}$-invariant symmetry breaking terms of Eq. (1). As will be seen, the SU(4) symmetric point is a rich multicritical point which unifies several emerging quantum orders. The nature of the quantum phase transition between these phases can then be determined within our approach and belongs to the Luttinger universality class [8] as the result of an exact U(1) self-duality symmetry at the transition [7].

The $S U(4)$ quantum critical point. The starting point of our approach is the existence of an SU(4) symmetric point in Eq. (1) which is obtained for $J_{11}=4 J_{1}$ and $J_{\perp}=J_{d}=J_{r r}=J_{d d}=0$ [9]. The resulting model can also be regarded as the SU(4) Heisenberg spin chain when the four states on a rung are identified with those of the fundamental representation 4 of SU(4). The latter model is Bethe-ansatz solvable [10] and displays an extended quantum criticality, which is characterized by SU(4) 1 conformal field theory (CFT) with central charge $c=3$ [11]. A simple description of this fixed point is provided by the conformal embedding $\mathrm{SU}(4)_{1} \sim \mathrm{SU}(2)_{2} \times \mathrm{SU}(2)_{2}$ with two $\mathrm{SU}(2) \mathrm{s}$ corresponding to independent rotations for the two chains. Since a single $\mathrm{SU}(2)_{2} \mathrm{CFT}$ is described by a triplet of real (Majorana) fermions, we may describe the critical properties of SU(4) $)_{1}$ fixed point by two triplets of rightand left-moving Majorana fermions $\xi_{R, L}^{a}$ and $\chi_{R, L}^{a}(a=1,2,3)$. This Majorana fermion description is extremely useful to understand the symmetry properties of model (1) in the close vicinity of the $S U(4)$ point as it has been exploited for the $\mathrm{SU}(2) \times \mathrm{SU}(2)$ spin-orbital chain [12, 13]. Moreover, the lattice discrete symmetries of model (1), i.e. one-step translation symmetry $\left(\mathscr{T}_{a_{0}}\right)$, time-reversal symmetry $(\mathscr{T})$, site-parity $\left(\mathscr{P}_{S}\right)$, and the permutation $\mathscr{P}_{12}$ of the two chains, are linearly represented in terms of the Majorana fermions. For instance, the translation symmetry is described by: $\xi_{R}^{a} \rightarrow-\xi_{R}^{a}$, $\chi_{R}^{a} \rightarrow-\chi_{R}^{a}$, whereas $\xi_{L}^{a}$ and $\chi_{L}^{a}$ are left unchanged under $\mathscr{T}_{a_{0}}$. These results lead us to write the most general low-energy effective field theory for the generalized two-leg spin ladder (1) which is invariant under the $\mathrm{SU}(2)$ spin rotational symmetry and the discrete symmetries $\mathscr{T}_{a_{0}} \times \mathscr{T} \times \mathscr{P}_{S} \times \mathscr{P}_{12}$ : 


$$
\begin{aligned}
& \mathscr{H}=\mathscr{H}_{0}+\left(g_{1}+g_{2}\right)\left[\left(\vec{\xi}_{R} \cdot \vec{\xi}_{L}\right)^{2}+\left(\vec{\chi}_{R} \cdot \vec{\chi}_{L}\right)^{2}\right]+\left(g_{1}-g_{2}\right)\left[\left(\vec{\xi}_{R} \cdot \vec{\chi}_{L}\right)^{2}+\left(\vec{\chi}_{R} \cdot \vec{\xi}_{L}\right)^{2}\right]+2\left(g_{3}+g_{4}\right)\left(\vec{\xi}_{R} \cdot \vec{\xi}_{L}\right)\left(\vec{\chi}_{R} \cdot \vec{\chi}_{L}\right) \\
& +2\left(-g_{3}+g_{4}\right)\left(\vec{\xi}_{R} \cdot \vec{\chi}_{L}\right)\left(\vec{\chi}_{R} \cdot \vec{\xi}_{L}\right)-\frac{g_{5}}{2}\left(\vec{\xi}_{R} \cdot \vec{\chi}_{R}\right)\left(\vec{\xi}_{L} \cdot \vec{\chi}_{L}\right)-i \frac{g_{6}}{2}\left(\vec{\xi}_{R} \cdot \vec{\chi}_{R}+\vec{\xi}_{L} \cdot \vec{\chi}_{L}\right),
\end{aligned}
$$

where $\mathscr{H}_{0}$ is the free-Hamiltonian for the Majorana fermions $\xi_{R, L}^{a}$ and $\chi_{R, L}^{a}$. No such strongly relevant mass terms for the Majorana fermions as $\vec{\xi}_{R} \cdot \vec{\xi}_{L}$ or $\vec{\chi}_{R} \cdot \vec{\chi}_{L}$ are allowed since they are odd under $\mathscr{T}_{a_{0}}$. The effective Hamiltonian (2) describes the low-energy properties of model 10 in the vicinity of the SU(4) point. In particular, using the continuum expressions of the spin operators $\mathbf{s}_{1,2, n}$ at the $\mathrm{SU}(4)_{1}$ fixed point found in Ref. [13], we have obtained the following identifications: $g_{1,2} \simeq$ $\left(J_{1} \pm J_{d}\right) / 2, g_{3,4} \simeq\left(J_{11} \pm J_{d d}\right) / 8, g_{5} \simeq J_{r r}$, and $g_{6} \simeq J_{\perp}$.

Order parameters and duality symmetries. Before investigating the infrared (IR) phases of the low-energy effective field theory (2), let us first discuss its symmetries and possible orders. The $\mathrm{SU}(4)_{1}$ fixed point Hamiltonian, i.e. $\mathscr{H}_{0}$, is invariant under chiral $\mathrm{SO}(2)$ rotations $\mathscr{R}_{r}(\theta), r=R, L$ on the Majorana fermions:

$$
\begin{aligned}
& \xi_{r}^{a} \rightarrow \xi_{r}^{a} \cos \theta / 2-\chi_{r}^{a} \sin \theta / 2 \\
& \chi_{r}^{a} \rightarrow \xi_{r}^{a} \sin \theta / 2+\chi_{r}^{a} \cos \theta / 2 .
\end{aligned}
$$

This rotation defines a first U(1) symmetry $\mathscr{U}_{\theta}=\mathscr{R}_{L}(\theta) \times$ $\mathscr{R}_{R}(\theta)$ which acts on the fields of the SU(4) $)_{1}$ CFT. Now we introduce a first set of order parameters-the staggered dimerization operator $\mathscr{O}_{\mathrm{SD}}=(-1)^{n}\left(\mathbf{s}_{1, n} \cdot \mathbf{s}_{1, n+1}-\mathbf{s}_{2, n} \cdot \mathbf{s}_{2, n+1}\right)$ and the scalar chirality order parameter [5, 6, 7]: $\mathscr{O}_{\mathrm{SC}}=$ $(-1)^{n}\left[\left(\mathbf{s}_{1, n}+\mathbf{s}_{2, n}\right) \cdot\left(\mathbf{s}_{1, n+1} \wedge \mathbf{s}_{2, n+1}\right)+(n \leftrightarrow n+1)\right] . \quad$ They have a simple continuum description in terms of the Majorana fermions: $\mathscr{O}_{\mathrm{SD}} \sim i\left(\vec{\xi}_{R} \cdot \vec{\xi}_{L}-\vec{\chi}_{R} \cdot \vec{\chi}_{L}\right)$ and $\mathscr{O}_{\mathrm{SC}} \sim$ $i\left(\vec{\xi}_{R} \cdot \vec{\chi}_{L}+\vec{\chi}_{R} \cdot \vec{\xi}_{L}\right)$ and from Eq. (3) we deduce that these order parameters transform as a doublet under $\mathscr{U}_{\theta}$ :

$$
\left(\begin{array}{c}
\mathscr{O}_{\mathrm{SD}} \\
\mathscr{O}_{\mathrm{SC}}
\end{array}\right) \rightarrow\left(\begin{array}{cc}
\cos \theta & -\sin \theta \\
\sin \theta & \cos \theta
\end{array}\right)\left(\begin{array}{c}
\mathscr{O}_{\mathrm{SD}} \\
\mathscr{O}_{\mathrm{SC}}
\end{array}\right)
$$

In particular, for $\theta=\pi / 2$, the two phases characterized by $\mathscr{O}_{\mathrm{SD}}$ and $\mathscr{O}_{\mathrm{SC}}$ are interchanged under $\mathscr{D}=\mathscr{U}_{\pi / 2}$ which can thus be viewed as a $\mathbb{Z}_{2}$ duality for a pair of order parameters. Remarkably, the duality symmetry $\mathscr{D}$ and the U(1) $\mathscr{U}_{\theta}$ transformation have a lattice interpretation which has been discovered previously and called spin-chirality symmetries by Momoi et al. [7]: $\mathscr{U}_{\theta}^{\text {lat }}=\exp \left[-i \theta \sum_{n}\left(\mathbf{s}_{1, n} \cdot \mathbf{s}_{2, n}-1 / 4\right)\right]$ and $\mathscr{D}=\mathscr{U}_{\theta=\pi / 2}^{\text {lat }}$.

Moreover, we can define two additional order parameters, expressed again as bilinears of Majorana fermions: $\mathscr{O}_{\mathrm{D}} \sim$ $i\left(\vec{\xi}_{R} \cdot \vec{\xi}_{L}+\vec{\chi}_{R} \cdot \vec{\chi}_{L}\right)$ and $\mathscr{O}_{\mathrm{RD}} \sim i\left(\vec{\xi}_{R} \cdot \vec{\chi}_{L}-\vec{\chi}_{R} \cdot \vec{\xi}_{L}\right)$ which are left invariant under the spin-chirality rotation $\mathscr{U}_{\theta}$ and are thus self-dual under $\mathscr{D}$. In fact, these order parameters are the continuum representation of the columnar dimerization operator
$\mathscr{O}_{\mathrm{D}}=(-1)^{n}\left(\mathbf{s}_{1, n} \cdot \mathbf{s}_{1, n+1}+\mathbf{s}_{2, n} \cdot \mathbf{s}_{2, n+1}\right)$ and the rung dimerization operator $\mathscr{O}_{\mathrm{RD}}=(-1)^{n} \mathbf{s}_{1, n} \cdot \mathbf{s}_{2, n}$. The latter order parameter describes a phase, with alternation of rung singlets and rung triplets, which has been found in some integrable twoleg spin ladder [14]. The second set of order parameters is closely related, in the continuum limit, to the existence of a second U(1) symmetry: $\tilde{\mathscr{U}}_{\theta}=\mathscr{R}_{L}(\theta) \times \mathscr{R}_{R}(-\theta)$. It leaves invariant the $\mathscr{O}_{\mathrm{SD}}$ and $\mathscr{O}_{\mathrm{SC}}$ order parameters whereas $\mathscr{O}_{\mathrm{D}}$ and $\mathscr{O}_{\mathrm{RD}}$ transform now as a doublet under $\tilde{\mathscr{U}}_{\theta}$ as in Eq. (4). A second $\mathbb{Z}_{2}$ duality, $\tilde{\mathscr{D}}=\tilde{\mathscr{U}}_{\pi / 2}$, can thus be considered as a transformation which maps the columnar dimerization onto the rung dimerization whereas the staggered dimerization and the scalar chirality are kept intact under $\tilde{\mathscr{D}}$. The $\mathrm{SU}(4)_{1}$ fixed point is therefore a rich multicritical point which unifies four different competing orders (dimerized $\mathscr{O}_{\mathrm{D}}, \mathscr{O}_{\mathrm{SD}}, \mathscr{O}_{\mathrm{RD}}$ and $\mathscr{T}$ breaking $\mathscr{O}_{\text {SC }}$. See Fig.1). The $\mathrm{SU}(4) \rightarrow \mathrm{SU}(2) \times \mathbb{Z}_{2}$ symmetry breaking perturbations will select one of these quantum orders as we are going to see now.

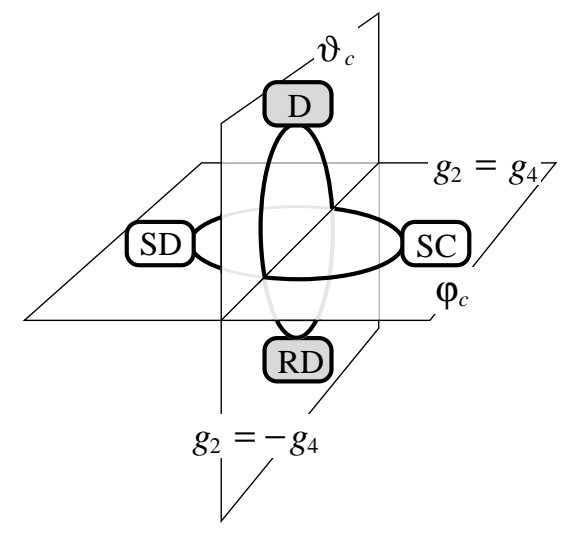

FIG. 1: Relationship between four orders. Note that on manifolds $g_{2}=g_{4}\left(g_{2}=-g_{4}\right)$ the system acquires U(1) symmetry under $\mathscr{U}_{\theta}$ $\left(\tilde{\mathscr{U}}_{\theta}\right)$ and order parameters form a doublet. Two angular fields $\varphi_{\mathrm{c}}$ and $\vartheta_{\mathrm{c}}$ characterize fluctuations within each doublet (see the text).

Renormalization Group $(R G)$ analysis. The next step of the approach is to perform a one-loop RG calculation to determine the nature of the IR phases of the low-energy effective Hamiltonian (2). First of all, the SU(4) model in Eq. (1), perturbed by a standard rung interaction $J_{\perp} \neq 0$ is Bethe-ansatz integrable [15]; for a small value of $J_{\perp}$, the gapless behavior of the $\mathrm{SU}(4)$ model with central charge $c=3$ extends up to a critical point $J_{\perp c}=4 J_{1}$ above which the standard gapped rung-singlet phase of the two-leg spin ladder appears. In the close vicinity of the SU(4) 1 quantum critical point, when $\left|\lambda_{i}\right| \ll 1$, we 
can thus forget the perturbation with coupling constant $g_{6}$ in Eq. (2). Thus we are left with marginal interactions and the one-loop RG equations read as follows:

$$
\begin{aligned}
& \dot{g}_{1}=g_{1}^{2}+g_{2}^{2}+5 g_{3}^{2}+g_{4}^{2} \\
& \dot{g}_{2}=2 g_{1} g_{2}+6 g_{3} g_{4}+g_{4} g_{5} \\
& \dot{g}_{3}=6 g_{1} g_{3}+2 g_{2} g_{4} \\
& \dot{g}_{4}=2 g_{1} g_{4}+6 g_{2} g_{3}+g_{2} g_{5} \\
& \dot{g}_{5}=-16\left(g_{1} g_{3}-g_{2} g_{4}\right) .
\end{aligned}
$$

We are now going to investigate the different gapped phases that emerge in the IR limit of the RG equations (5). First of all, it is important to note that the interaction part of the Hamiltonian with $g_{5,6}=0$ can be recasted as

$$
\mathscr{H}_{\mathrm{int}}=-\lambda_{\mathrm{SD}} \mathscr{O}_{\mathrm{SD}}^{2}-\lambda_{\mathrm{SC}} \mathscr{O}_{\mathrm{SC}}^{2}-\lambda_{\mathrm{D}} \mathscr{O}_{\mathrm{D}}^{2}-\lambda_{\mathrm{RD}} \mathscr{O}_{\mathrm{RD}}^{2}
$$

where the couplings $\lambda_{\mathrm{SD}}$ etc. are functions of $g_{1}, \ldots, g_{4}$ and Eq. (6) describes the competition between the quantum orders introduced previously. Then we apply an ansatz, proposed by Lin et al. [16] in the context of the half-filled two-leg Hubbard ladder, that the IR asymptotics of Eq. (5) is described by: $g_{i}(t)=r_{i} /\left(t_{0}-t\right)$, where $t$ is the RG time and $t_{0}$ marks the crossover point where the weak-coupling perturbation breaks down. The coefficients $r_{i}$ indicate the symmetric rays which attract the RG flow in the IR limit and define the different strong coupling phases of the problem. A first symmetric ray, $\left(r_{1}, r_{2}, r_{3}, r_{4}, r_{5}\right)=(1 / 8,1 / 8,-1 / 8,-1 / 8,0)$, is described by the low-energy effective field theory [13]:

$$
\mathscr{H}_{\text {eff }}^{(1)}=\mathscr{H}_{0}+\lambda_{\mathrm{SD}}^{*}\left(\vec{\xi}_{R} \cdot \vec{\xi}_{L}-\vec{\chi}_{R} \cdot \vec{\chi}_{L}\right)^{2}
$$

with $\lambda_{\mathrm{SD}}^{*}>0$ so that, after a transformation $\vec{\chi}_{R} \rightarrow-\vec{\chi}_{R}$, the Hamiltonian (7) takes the form of a SO(6) Gross-Neveu (GN) model which is an integrable field theory with a spectral gap [17]. The nature of the resulting strong-coupling phase can be elucidated by observing that the interacting part of Eq. (7) is simply: $\mathscr{H}_{\mathrm{int}}=-\lambda_{\mathrm{SD}}^{*} \mathscr{O}_{\mathrm{SD}}^{2}$, so that $\left\langle\mathscr{O}_{\mathrm{SD}}\right\rangle= \pm \Delta_{0} \neq 0$ in the IR limit. A staggered dimerized phase, as in the $\mathrm{SU}(2) \times$ $\mathrm{SU}(2)$ spin-orbital chain [12, 13], is thus stabilized and breaks spontaneously the translation symmetry. A second symmetric ray, $\left(r_{1}, r_{2}, r_{3}, r_{4}, r_{5}\right)=(1 / 8,-1 / 8,-1 / 8,1 / 8,0)$ is described by

$$
\mathscr{H}_{\text {eff }}^{(2)}=\mathscr{H}_{0}+\lambda_{\mathrm{SC}}^{*}\left(\vec{\xi}_{R} \cdot \vec{\chi}_{L}+\vec{\chi}_{R} \cdot \vec{\xi}_{L}\right)^{2}
$$

Upon the chiral transformation $\vec{\chi}_{R} \leftrightarrow \vec{\xi}_{R}$, the Hamiltonian (8) is mapped onto the $\mathrm{SO}(6) \mathrm{GN}$ model. The interacting part of Eq. 8 is written now as $\mathscr{H}_{\text {int }}=-\lambda_{\mathrm{SC}}^{*} \mathscr{O}_{\mathrm{SC}}^{2}$, and thus implies $\left\langle\mathscr{O}_{\mathrm{SC}}\right\rangle= \pm \Delta_{0} \neq 0$, i.e. the emergence of the scalar chirality phase. which breaks spontaneously the time-reversal symmetry. At this point, it is worth observing that the Hamiltonian (7) and (8) are interchanged under the duality symmetry $\mathscr{D}=\mathscr{U}_{\theta=\pi / 2}$ on the Majorana fermions. In this respect, the approach used here gives the field-theoretical interpretation to the spin-chirality duality and the appearance of the long-range (staggered) scalar chiral order in the phase diagram of Eq. (1) which was first pointed out in Ref. [7].

Finally, in a similar way, there are two more symmetric rays where the $\mathrm{SO}(6)$ symmetry is restored in the IR limit: $\left(r_{1}, r_{2}, r_{3}, r_{4}, r_{5}\right)=(1 / 8,1 / 8,1 / 8,1 / 8,0)$ and $\left(r_{1}, r_{2}, r_{3}, r_{4}, r_{5}\right)=(1 / 8,-1 / 8,1 / 8,-1 / 8,0)$. They correspond respectively to the stabilization of the columnar dimerization $\left(\mathscr{O}_{\mathrm{D}}\right)$ and the rung dimerization $\left(\mathscr{O}_{\mathrm{RD}}\right)$ which are interchanged now under the second duality $\tilde{\mathscr{D}}$. In summary, the four phases, $\mathscr{O}_{\mathrm{SD}}, \mathscr{O}_{\mathrm{SC}}, \mathscr{O}_{\mathrm{D}}, \mathscr{O}_{\mathrm{RD}}$, related two by two through the duality symmetries $\mathscr{D}$ and $\tilde{\mathscr{D}}$, are the different gapped phases of the problem which are characterized by an $\mathrm{SO}(6)$ symmetry restoration in the IR limit.

Quantum phase transition. In addition to these $\mathrm{SO}(6)$ symmetric rays, there are special manifolds where the RG equations (5) display also an larger symmetric behavior. On the two manifolds $g_{2}= \pm g_{4}$, the $\mathrm{SU}(2) \times \mathbb{Z}_{2}$ symmetric model (2) acquires a larger continuous symmetry $\mathrm{SU}(2) \times \mathrm{U}(1)$, being invariant under arbitrary rotations $\mathscr{U}_{\theta}$ and $\tilde{\mathscr{U}}_{\theta}$ respectively (see Fig.1). Within these self-dual manifolds, the RG flow is attracted in the IR limit towards two different asymptotes: $\left(r_{1}, r_{2}, r_{3}, r_{4}, r_{5}\right)=(1 / 6,0, \mp 1 / 6,0, \pm 4 / 9)$. Along the first line $\left(r_{3}=-r_{1}\right)$, the interacting part of the low-energy self-dual theory takes the form:

$$
\mathscr{H}_{\mathrm{eff}}^{\mathrm{int}}=-\lambda^{*}\left(\mathscr{O}_{\mathrm{SD}}^{2}+\mathscr{O}_{\mathrm{SC}}^{2}\right)-\frac{4 \lambda^{*}}{3}\left(\vec{\xi}_{R} \cdot \vec{\chi}_{R}\right)\left(\vec{\xi}_{L} \cdot \vec{\chi}_{L}\right),
$$

which describes the competition between the staggered dimerization and scalar chirality orders, i.e. governs the nature of the quantum phase transition between these two phases. Similarly, the second asymptote accounts for the competition between the columnar dimerization and the rung dimerization. The emerging effective field theory (9) displays a larger symmetry than the $\mathrm{U}(1) \times \mathrm{SU}(2)$ symmetry of the initial manifold $g_{2}=g_{4}$. On the one hand, model (9) turns out to be invariant not only under $\tilde{\mathscr{U}}_{\theta}$ but also under a larger $\mathscr{U}_{\theta} \times \tilde{\mathscr{U}}_{\theta}$ symmetry (Note that at the lattice level, $\tilde{\mathscr{U}}_{\theta}$ may be broken by umklapp interactions). On the other hand, it is also invariant under a hidden SU(3) symmetry. A way to reveal this last symmetry is to combine the six Majorana fermions into three Dirac fermions: $\Psi_{a R, L}=\left(\xi_{R, L}^{a}+i \chi_{R, L}^{a}\right) / \sqrt{2}$. The $\mathscr{U}_{\theta}$ and $\tilde{\mathscr{U}}_{\theta}$ rotations then acquire a simple meaning in terms of these Dirac fermions since they act on them as charge U(1) chiral symmetries: $\Psi_{a R, L} \rightarrow e^{i \theta / 2} \Psi_{a R, L}$ for $\mathscr{U}_{\theta}$ and $\Psi_{a R, L} \rightarrow e^{ \pm i \theta / 2} \Psi_{a R, L}$ for $\tilde{\mathscr{U}}_{\theta}$. The self-duality symmetry $\mathscr{U}_{\theta} \times \tilde{\mathscr{U}}_{\theta}$ of Eq. (9) is described thus as an $\mathrm{U}(1)_{\mathrm{R}} \times \mathrm{U}(1)_{\mathrm{L}}$ symmetry on the Dirac fermions. In addition, we can see that the order parameters $\mathscr{O}_{\mathrm{SD}}$ and $\mathscr{O}_{\mathrm{SC}}$ have also a simple interpretation as Cooper pairs by taking a combination $\mathscr{O}_{\mathrm{SD}}+i \mathscr{O}_{\mathrm{SC}} \sim \vec{\Psi}_{R} \cdot \vec{\Psi}_{L}$. The role of these pseudo-charge degrees freedom, introduced by the spin-chirality $U(1)$ symmetry, becomes manifest with the help of a bosonization of the Dirac fermions. To this end, we define three right-left moving bosonic fields $\varphi_{a R, L}$ such as: $\Psi_{a R, L} \sim \exp \left[ \pm i \sqrt{4 \pi} \varphi_{a R, L}\right]$, and switch to a basis where the 
pseudo-charge degrees of freedom single out:

$$
\begin{aligned}
\varphi_{c R, L} & =\frac{1}{\sqrt{3}}\left(\varphi_{1 R, L}+\varphi_{2 R, L}+\varphi_{3 R, L}\right) \\
\varphi_{s R, L} & =\frac{1}{\sqrt{2}}\left(\varphi_{1 R, L}-\varphi_{2 R, L}\right) \\
\varphi_{f R, L} & =\frac{1}{\sqrt{6}}\left(\varphi_{1 R, L}+\varphi_{2 R, L}-2 \varphi_{3 R, L}\right) .
\end{aligned}
$$

In terms of these new fields, the low-energy Hamiltonian (9) exhibits a "spin"(SU(3))-“charge"(U(1)) separation $\mathscr{H}_{\text {eff }}=$ $\mathscr{H}_{\mathrm{c}}+\mathscr{H}_{\mathrm{s}}$, with $\left[\mathscr{H}_{\mathrm{c}}, \mathscr{H}_{\mathrm{s}}\right]=0$. The charge degrees of freedom are described by the Tomonaga-Luttinger Hamiltonian:

$$
\mathscr{H}_{\mathrm{c}}=\frac{v}{2}\left[\left(\partial_{x} \varphi_{\mathrm{c}}\right)^{2}+\left(\partial_{x} \vartheta_{\mathrm{c}}\right)^{2}\right]
$$

where $\varphi_{\mathrm{c}}=\varphi_{\mathrm{c} R}+\varphi_{\mathrm{c} L}$ is the total charge bosonic field and $\vartheta_{\mathrm{c}}$ is its dual field. The Hamiltonian $\mathscr{H}_{s}$ for the remaining degrees of freedom can be recasted in a fully $\mathrm{SU}(3)$ symmetric form in terms of the chiral SU(3) $)_{1}$ currents $\mathscr{J}_{R, L}^{A}$ defined from the two bosonic fields $\varphi_{s, f R, L}$ :

$$
\mathscr{H}_{S}=\frac{\pi v}{2} \sum_{A=1}^{8}\left(\mathscr{J}_{R}^{A} \mathscr{J}_{R}^{A}+\mathscr{J}_{L}^{A} \mathscr{J}_{L}^{A}\right)+g^{*} \sum_{A=1}^{8} \mathscr{J}_{R}^{A} \mathscr{J}_{L}^{A}
$$

The latter model is the SU(3) GN model which is a massive $\left(g^{*}>0\right)$ integrable field theory [18]. A spectral gap is thus formed by the interactions in the "spin" sector and the low-lying excitations are known, from the exact solution [18], to be massive SU(3) spinons and antispinons. The low-energy physics of 9 is dominated by the gapless spinsinglet fluctuations 11 of the "charge" degrees of freedom which stems from the remarkable U(1)-symmetry $\left(\mathscr{U}_{\theta}\right)$ of Eq. (9). Therefore we may conclude that the quantum phase transition between the staggered dimerized- and scalar chirality phases belongs to the $c=1$ Luttinger-liquid universality class. The physical properties at the transition can also be determined within our approach. At the transition, all order parameters have zero expectation values: $\left\langle\mathscr{O}_{\mathrm{SD}}\right\rangle=\left\langle\mathscr{O}_{\mathrm{SC}}\right\rangle=$ $\left\langle\mathscr{O}_{\mathrm{D}}\right\rangle=\left\langle\mathscr{O}_{\mathrm{RD}}\right\rangle=0$. The first doublet $\mathscr{O}_{\mathrm{SD}}$ and $\mathscr{O}_{\mathrm{SC}}$ has a fixed modulus and correlation functions decaying as $x^{-2 / 3}$, i.e. has long-range coherence, whereas the second one $\mathscr{O}_{\mathrm{D}}$ and $\mathscr{O}_{\mathrm{RD}}$ is exponentially decaying due to strong quantum fluctuations. Now it is straightforward to discuss the effect of a small deviation from the self-dual manifold (9) by switching on the perturbation: $\mathscr{V}=\varepsilon\left(\mathscr{O}_{\mathrm{SD}}^{2}-\mathscr{O}_{\mathrm{SC}}^{2}\right),|\varepsilon| \ll 1$ which breaks in particular the $\mathscr{U}_{\theta}$ symmetry of model (9). This small symmetry-breaking perturbation does not close the spin gap but the charge Hamiltonian 11 acquire now a 'pinning' term $\mathscr{V}_{c} \simeq-\varepsilon \cos \left(\sqrt{16 \pi / 3} \varphi_{c}\right)$ and becomes equivalent to a quantum sine-Gordon model. The interaction has scaling dimension $\Delta=4 / 3<2$ so that the perturbation opens a charge gap; for $\varepsilon<0$ (respectively $\varepsilon>0$ ), the staggered dimerization (respectively scalar chirality) order is stabilized by the small symmetry-breaking term. The same argument applies to the second ray $\left(r_{1}=r_{3}\right)$ as well after the replacement $\varphi_{c} \leftrightarrow \vartheta_{c}$ and describes the competition between $\mathscr{O}_{\mathrm{D}}$ and $\mathscr{O}_{\mathrm{RD}}$.

In summary, we have shown, in the continuum approach, that four different gapped phases around the SU(4) multicritical point are unified by the hidden $\mathbb{Z}_{2}$ symmetries $\mathscr{D}$ and $\tilde{\mathscr{D}}$. The spin-chirality $U(1) \mathscr{U}_{\theta}$ symmetry plays an essential role on the self-dual manifold and as a consequence a second-order phase transition which separates the staggered dimerized- and scalar chirality phases is characterized by a $c=1$ Luttingerliquid fixed point. This point was unclear in the preceding papers mainly because of numerical limitations. On the basis of this fact, we explained how an exotic phase with a broken time-reversal symmetry is stabilized. Moreover, by a mapping onto a low-energy effective theory, we have revealed another hidden relationship between columnar-dimer phase and rung-dimer phase together with a corresponding U(1) symmetry $\tilde{\mathscr{U}}_{\theta}$. Finally, a similar unifying approach based on an U(4) symmetry can also be performed to describe the exotic phases and transitions in doped generalized two-leg ladders as it will be discussed elsewhere.

The authors are very grateful to E. Boulat for illuminating discussions. They would like also to thank P. Azaria, A. Läuchli, T. Momoi, E. Orignac for very useful discussions.

[1] D. J. Thouless, Proc. Phys. Soc. London 86, 893 (1965); M. Roger, J. H. Hetherington, and J. M. Delrieu, Rev. Mod. Phys. 55, 1 (1983).

[2] M. Matsuda et al., Phys. Rev. B 62, 8903 (2000).

[3] A. Paramekanti, L. Balents, and M. P. A. Fisher, Phys. Rev. B 66, 054526 (2002).

[4] X. G. Wen, F. Wilczek, and A. Zee, Phys.Rev. B 39, 11413 (1989).

[5] A. Läuchli, G. Schmid, and M. Troyer, Phys. Rev. B 67, 100409(R) (2003).

[6] T. Hikihara, T. Momoi, and X. Hu, Phys. Rev. Lett. 90, 087204 (2003).

[7] T. Momoi, T. Hikihara, M. Nakamura, and X. Hu, Phys. Rev. B 67, 174410 (2003).

[8] F. D. M. Haldane, Phys. Rev. Lett. 45, 1358 (1980).

[9] Y. Q. Li et al., Phys. Rev. Lett. 81, 3527 (1998); Y. Yamashita, N. Shibata, and K. Ueda, Phys. Rev. B 58, 9114 (1998).

[10] B. Sutherland, Phys. Rev. B 12, 3795 (1975).

[11] I. Affleck, Nucl. Phys. B 305, 582 (1988).

[12] A. A. Nersesyan and A. M. Tsvelik, Phys. Rev. Lett. 78, 3939 (1997).

[13] P. Azaria et al., Phys. Rev. Lett. 83, 624 (1999); P. Azaria, E. Boulat, and P. Lecheminant, Phys. Rev. B 61, 12112 (2000).

[14] Y. Wang, Int. J. Mod. Phys. B 13, 3323 (1999); G. Albertini, Phys. Rev. B 64, 094416 (2001).

[15] Y. Wang, Phys. Rev. B 60, 9236 (1999).

[16] H.-H. Lin, L. Balents, and M. P. A. Fisher, Phys. Rev. B 58, 1794 (1998).

[17] A. A. Zamolodchikov and A. B. Zamolodchikov, Ann. Phys. (N.Y.) 120, 253 (1979).

[18] N. Andrei and J. H. Lowenstein, Phys. Lett. B 90, 106 (1980). 